\title{
Association between perioperative glucocorticoids and cancer metastasis and survival in patients undergoing radical cystectomy for urothelial carcinoma of the bladder: A single- center retrospective study
}

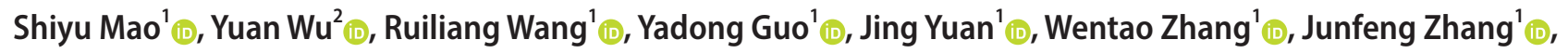 \\ Yang Yan ${ }^{1}$, ${ }^{1}$ Xudong Yao ${ }^{1}$ (i) \\ 'Department of Urology, Shanghai Tenth People's Hospital, Tongji University School of Medicine, Shanghai, ${ }^{2}$ Department of Urology, Shanghai Tenth People's Hospital, \\ Anhui Medical University, Hefei, China
}

Purpose: Perioperative glucocorticoids have the potential to increase the risk of tumor metastasis. However, the relationship between perioperative glucocorticoids and oncologic outcomes remains controversial. The present study was undertaken to evaluate the association of perioperative glucocorticoids with clinicopathologic outcomes following radical cystectomy (RC).

Materials and Methods: We screened and included 185 patients who underwent radical surgery for bladder cancer in our center between 2009 and 2018. The Kaplan-Meier method was applied, and a log-rank test was used to estimate differences in metastasis-free survival (MFS) and overall survival (OS) between the groups. Multivariate Cox proportional hazards regression models were used to analyze any association of glucocorticoids with clinical outcomes.

Results: A total of 76 (41.1\%) patients received perioperative glucocorticoids. Median postoperative follow-up was 2.0 years. Kaplan-Meier survival curve indicated that the glucocorticoids group was significantly associated with increased distant MFS ( $p=0.008$ ) but not with OS. In the multivariate analysis, no significant differences were observed for MFS between the groups. Interestingly, when the variable of blood transfusion was excluded from the multivariate analysis model, we found that patients receiving glucocorticoids were independently associated with worse MFS (hazard ratio, 1.790; $p=0.030$ ). Furthermore, the partial correlation analysis showed a significant positive correlation between perioperative glucocorticoids and blood transfusion ( $r=0.604$, $\mathrm{p}<0.001)$. In the nontransfusion subgroup, propensity score matching showed that patients receiving glucocorticoids had a higher risk of distant metastasis.

Conclusions: Perioperative glucocorticoids were associated with a higher rate of distant metastasis in patients undergoing RC for bladder cancer.

Keywords: Glucocorticoids; Neoplasm metastasis; Surgery; Urinary bladder neoplasms 


\section{INTRODUCTION}

Urinary bladder cancer $(\mathrm{BC})$ is the tenth most common cancer in the world [1]. The standard treatment for high-risk nonmuscle invasive and localized muscle invasive bladder cancer (MIBC) is radical cystectomy (RC). Several studies have reported that surgical procedures increase the risk of tumor cells entering the bloodstream, which may lead to increased risk of clinical recurrence and metastasis [2,3]. Perioperative intervention factors, including anesthesia and drug use, may affect the survival of circulating tumor cells and thus have an impact on tumor outcomes [4]. It is well known that glucocorticoids (GCs) have anti-inflammatory and immunosuppressive effects, which might potentially strengthen the inhibition of perioperative antitumor immunity and facilitate the survival and metastasis of tumor cells $[5,6]$. In addition, recent preclinical studies have found that the activation of GC receptor (GR) is involved in tumorigenesis, metastasis, and chemoresistance of breast cancer [7,8]. Moreover, GR activation is also considered to be one of the mechanisms of antiandrogen receptor therapy resistance in prostate cancer [9]. These results suggest that GCs potentially increase heterogeneity and metastasis. GCs are commonly used in anesthetic practice for prophylaxis against postoperative nausea and vomiting, and they can also be used to prevent allergies before transfusion. Despite their widespread use, few clinical studies have evaluated the effects of perioperative GCs on oncological outcomes, and the results obtained are conflicting.

Although RC for BC combined with postoperative adjuvant therapy for MIBC has been performed, long-term clinical outcomes after surgery are not satisfactory, and the 5-year overall survival (OS) is only approximately 50\% [10]. Morbidity and mortality associated with $\mathrm{BC}$ are mainly caused by distant metastases [11,12]. Therefore, it is necessary to investigate follow-up data on patients undergoing radical surgery for $\mathrm{BC}$ to define the association between perioperative administration of systemic GCs and oncological outcomes.

\section{MATERIALS AND METHODS}

\section{Study population}

This study was approved by the Institutional Review Board of Shanghai Tenth People's Hospital (approval number: SHSY-IEC-4.1/19-120/01). Written informed consent was obtained from all the enrolled patients prior to treatment. A total of 248 patients with Ta-T4 stage urothelial carcinoma, who underwent RC between January 2009 and October 2018 at the Shanghai Tenth People's Hospital, were retrospectively evaluated in the study. The exclusion criteria were incomplete clinical data, lost to follow-up, having two concomitant tumors, nontransitional cell carcinoma, and preoperative distal metastases. Clinical and pathological data were collected from medical records. Patient characteristics included age and sex, history of hypertension and diabetes, history of postoperative bladder instillation, TNM staging, differential grading, number of tumors, size of the largest tumor, and the presence of coexisting carcinoma in situ (CIS).

Metastasis-free survival (MFS) was calculated as the period from the date of operation to the date of distant metastasis or death from tumor. OS was calculated as the period from the date of operation to the date of death from any cause. Inpatient medical records and anesthesia notes were evaluated, and patients who had been given intravenous GCs postoperatively and/or intraoperatively were assigned to the GC group. Reasons for patients receiving GCs perioperatively included intraoperative administration for nausea and vomiting, blood transfusion, allergy, and high fever. Perioperative period is defined as from one week before surgery to two weeks after surgery. The patients were usually given 5 or $10 \mathrm{mg}$ of dexamethasone sodium phosphate injection or $40 \mathrm{mg}$ of methylprednisolone sodium succinate for injection, as required for clinical treatment.

\section{Statistical analyses}

Patients who received GC and those who did not were compared using Student's t-test for continuous variables and the chi-square test for categorical variables. All p-values less than 0.05 were considered statistically significant. KaplanMeier survival analysis and the log-rank test were used to evaluate the univariate associations between GC usage and survival outcomes. Univariate Cox regression analysis was used to screen potential risk factors associated with metastasis and mortality after BC surgery. Variables that were introduced in multivariate analysis were those that were associated $(p<0.05)$ with MFS or OS in univariate analyses. A multivariate analysis using Cox regression models was used to determine the association between potential risk factors and survival. We also performed a correlation analysis to estimate the correlation between GC and blood transfusion. Propensity score matching was used to further evaluate the relationship between GC and MFS in the nontransfusion subgroup. Variables were adjusted to factors including all baseline parameters, and the score was calculated with logistic regression analysis. A maximize execution performance algorithm was used to identify the optimally matched groups with a match tolerance of 0.1 during matching simi- 


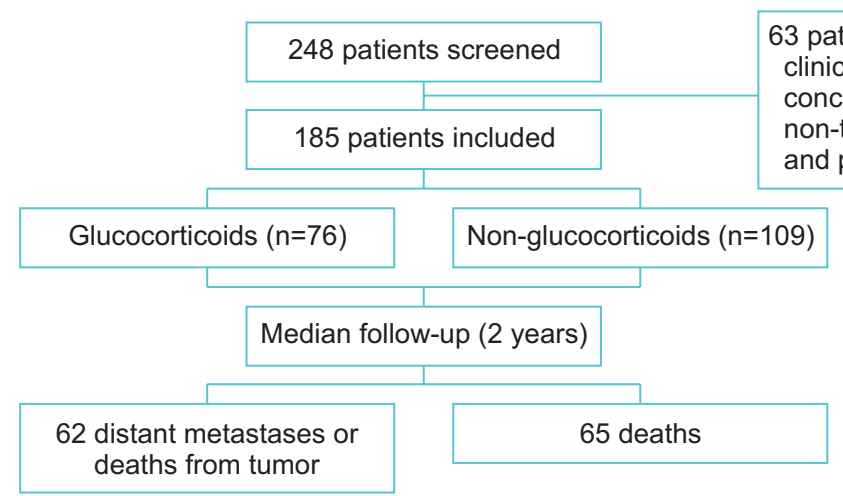

lar propensity scores. All statistical analyses were conducted with IBM SPSS Statistics version 24.0 (IBM Corp., Armonk, NY, USA).

\section{RESULTS}

According to the inclusion and exclusion criteria, 185 patients were included in the study (Fig. 1). These 185 patients included 24 females and 161 males, with a mean age of 66.9 years (range, 32 to 87 years). During the perioperative period, 76 patients received GCs. Of these 76 patients, 58 patients received GCs intraoperatively. Twelve patients were administered $10 \mathrm{mg}, 54$ patients were administered 10 to $20 \mathrm{mg}$, and 10 patients were administered more than $20 \mathrm{mg}$ GCs. The clinicopathological baseline data of the two groups are shown in Table 1. There were no significant differences in clinical parameters between the two groups, except for tumor number and blood transfusion rate $(\mathrm{p}=0.028$ and $\mathrm{p}<0.001$, respectively). Correlation analysis showed a significant association between GC usage and blood transfusions $(r=0.604$, $\mathrm{p}<0.001$ ) (Supplementary Table 1). The overall metastasis rate was 33.5\% (62/185), and patients who received GCs had higher risk of metastasis than those who did not (50.0\% [38/76] vs. $22.0 \%[24 / 109]$, $p<0.001)$.

\section{The impact of GCs on survival in BC surgery}

The median follow-up time was 2 (interquartile range, 1.0-4.2) years. Kaplan-Meier survival analysis showed that GCs significantly increased the risk of metastasis for MFS in $\mathrm{BC}(\mathrm{p}=0.008)$ (Fig. $2 \mathrm{~A})$, but not for $\mathrm{OS}(\mathrm{p}=0.449)$ (Fig. $2 \mathrm{~B})$. In univariate analyses, GCs (hazard ratio [HR], 1.980; 95\% confidence interval [CI], 1.181-3.321; $\mathrm{p}=0.010$ ) as well as age, T stage, nodal status, and blood transfusion were associated with MFS (Table 2). However, GCs showed no significant association with OS (HR, 1.185; 95\% CI, 0.721-1.946; $\mathrm{p}=0.503$ ). In addition, according to the dose of perioperative GCs, GCs (HR, 1.274; 95\% CI, 1.006-1.612; p=0.044) was also associated with MFS, but not for OS (HR, 1.053; 95\% CI, 0.831-1.334; $\mathrm{p}=0.672$ ). Furthermore, multivariate analyses were used to assess the association between perioperative GC administration and MFS. The results, however, revealed no association between perioperative GC use and MFS (HR, 1.462; 95\% CI, 0.754-2.836; $p=0.261$ ) even after adjusting for age, $\mathrm{T}$ stage, nodal status, and blood transfusion. Interestingly, when the variable of blood transfusion was excluded from the multivariate analysis model, we found that patients receiving GCs were independently associated with worse MFS (HR, 1.790; 95\% CI, 1.058-3.028; p=0.030). Again, when the variable of receiving GCs was excluded from the multivariate analysis model, we found that patients receiving blood transfusion were independently associated with worse MFS (HR, 1.751; 95\% CI, 1.036-2.960; $p=0.036$ ) (Table 3). Furthermore, the multivariate analysis model showed no significant association between GCs and OS (HR, 0.646; 95\% CI, 0.344-1.211; p=0.173). Age, nodal status, and perioperative blood transfusion were significantly associated with OS $(p<0.05)$. When the variable of blood transfusion was excluded from the multivariate analysis model, GCs did not significantly influence the OS (HR, 0.972; 95\% CI, 0.585-1.616; p=0.914).

\section{Subgroup analyses for association between GCs and MFS in the nontransfusion subgroup}

There were 103 patients without perioperative blood transfusion. Of these 103 patients, 15 patients received GCs. Propensity score matching was used to further evaluate the relationship between GC and MFS in the nontransfusion subgroup. After matching for all baseline parameters, the subgroup analyses showed no association between dexamethasone administration and OS in the nontransfusion subgroup ( $p=0.905$ ). However, GCs showed a nonsignificant tendency to increase the risk of metastasis in $\mathrm{BC}(\mathrm{HR}, 2509$; 95\% CI, 0.725-8.683; p=0.132) (Fig. 2C). 
Table 1. Clinicopathologic characteristics of the study cohort

\begin{tabular}{|c|c|c|c|c|}
\hline Variable & Total $(n=185)$ & Non-glucocorticoid $(n=109)$ & Glucocorticoid ( $n=76)$ & $p$-value \\
\hline Age (y) & $66.9(32-87)$ & $66.3(32-87)$ & $67.7(37-87)$ & $0.350^{\mathrm{a}}$ \\
\hline Sex & & & & 0.950 \\
\hline Female & $24(13.0)$ & $14(12.8)$ & $10(13.2)$ & \\
\hline Male & $161(87.0)$ & $95(87.2)$ & $66(86.8)$ & \\
\hline Pathologic T stage & & & & 0.165 \\
\hline$\leq \mathrm{T} 1$ & $83(44.9)$ & $48(44.0)$ & $35(46.1)$ & \\
\hline $\mathrm{T} 2$ & $36(19.5)$ & $21(19.3)$ & $15(19.7)$ & \\
\hline T3 & $37(20.0)$ & $18(16.5)$ & $19(25.0)$ & \\
\hline T4 & $29(15.7)$ & $22(20.2)$ & $7(9.2)$ & \\
\hline Nodal status & & & & 0.836 \\
\hline Positive & $28(15.1)$ & $16(14.7)$ & $12(15.8)$ & \\
\hline Negative & $157(84.9)$ & $93(85.3)$ & $64(84.2)$ & \\
\hline Tumor grade & & & & 0.210 \\
\hline Low & $9(4.9)$ & $3(2.8)$ & $6(7.9)$ & \\
\hline High & $176(95.1)$ & $106(97.2)$ & $70(92.1)$ & \\
\hline Tumor size $(\mathrm{cm})$ & & & & 0.984 \\
\hline$<3$ & $68(36.8)$ & $40(36.7)$ & $28(36.8)$ & \\
\hline$\geq 3$ & $117(63.2)$ & $69(63.3)$ & $48(63.2)$ & \\
\hline Number of tumors & & & & 0.028 \\
\hline Single & $76(41.1)$ & $52(47.7)$ & $24(31.6)$ & \\
\hline Multiple & $109(58.9)$ & $57(52.3)$ & $52(68.4)$ & \\
\hline Prior recurrence status & & & & 0.651 \\
\hline Primary & $101(54.6)$ & $58(53.2)$ & $43(56.6)$ & \\
\hline Recurrence & $84(45.4)$ & $51(46.8)$ & $33(43.4)$ & \\
\hline Concomitance of CIS & & & & 0.097 \\
\hline Yes & $13(7.0)$ & $11(10.1)$ & $2(2.6)$ & \\
\hline No & $172(93.0)$ & $98(89.9)$ & $74(97.4)$ & \\
\hline Intraoperative blood transfusion & & & & $<0.001$ \\
\hline Yes & $82(44.3)$ & $21(19.3)$ & $61(80.3)$ & \\
\hline No & $103(55.7)$ & $88(80.7)$ & $15(19.7)$ & \\
\hline Receipt of adjuvant therapy & $32(17.3)$ & $16(14.7)$ & $16(21.1)$ & 0.259 \\
\hline
\end{tabular}

Values are presented as mean (range) or number (\%).

CIS, carcinoma in situ.

${ }^{\text {a}}$ Student's t-test.

\section{DISCUSSION}

In the present study, we found that perioperative GC may potentially increase the risk of metastasis of patients after BC surgery. First, our results showed that the GC group had a significantly higher percentage of patients who received blood transfusion. Furthermore, we found a significant positive correlation between the use of GC and blood transfusion. Next, the results of the Kaplan-Meier survival and univariate analyses suggested that patients receiving GCs were associated with worse MFS, but not with OS, which may be attributed to nontumor causes of death after cancer surgery. However, after adjusting for all risk factors, including blood transfusion, the results of the multivariate analysis revealed no association between perioperative GC use and MFS. The overall Cox model also showed a similar result for blood transfusion, which is inconsistent with published literature [13,14]. Considering the significant positive correlation between GC and blood transfusion, we speculate that two prognostic factors may interfere with each other in the prognostic analysis. There may be a mutual relationship between the influence of GC and blood transfusion on MFS in the multivariate analysis model. Therefore, when the variable of blood transfusion or GCs was excluded from the multivariate analysis model, we found that patients receiving GCs or blood transfusion were independently associated with worse MFS. After matching for all baseline parameters using the propensity score matching method in the non- 

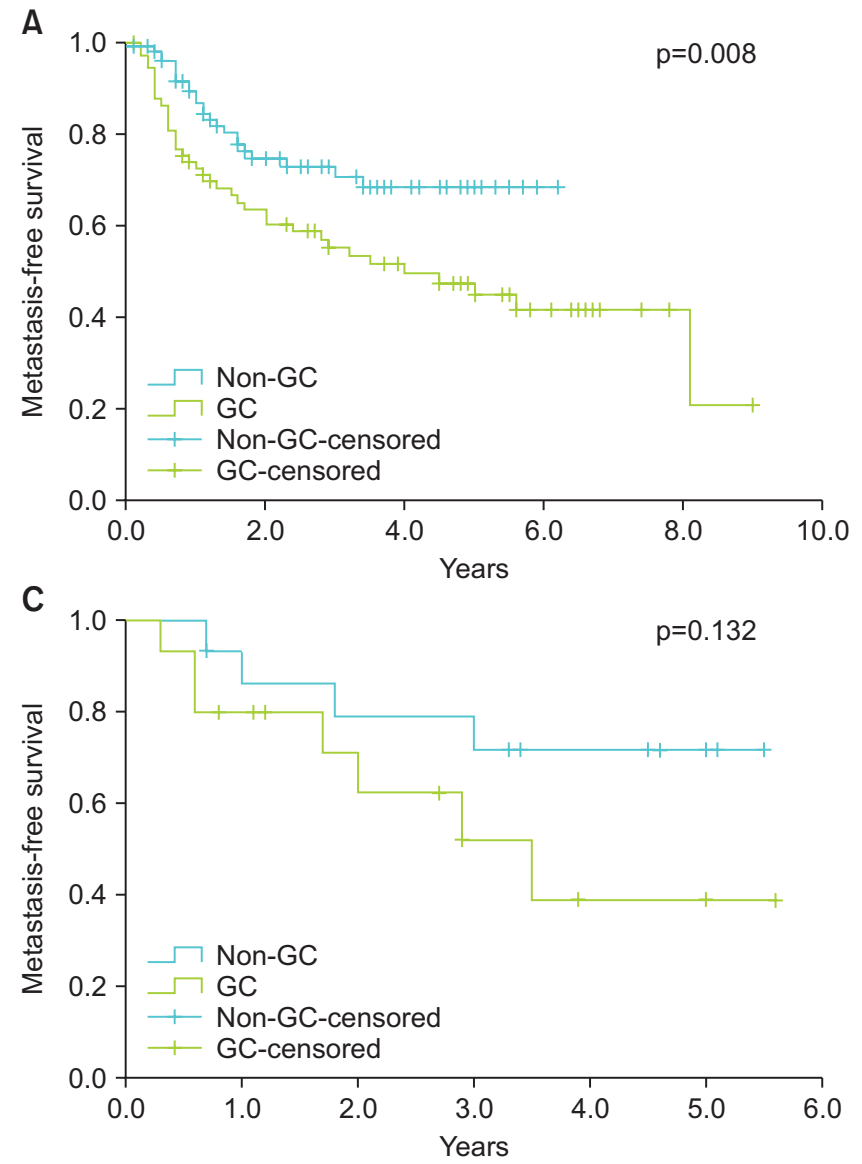

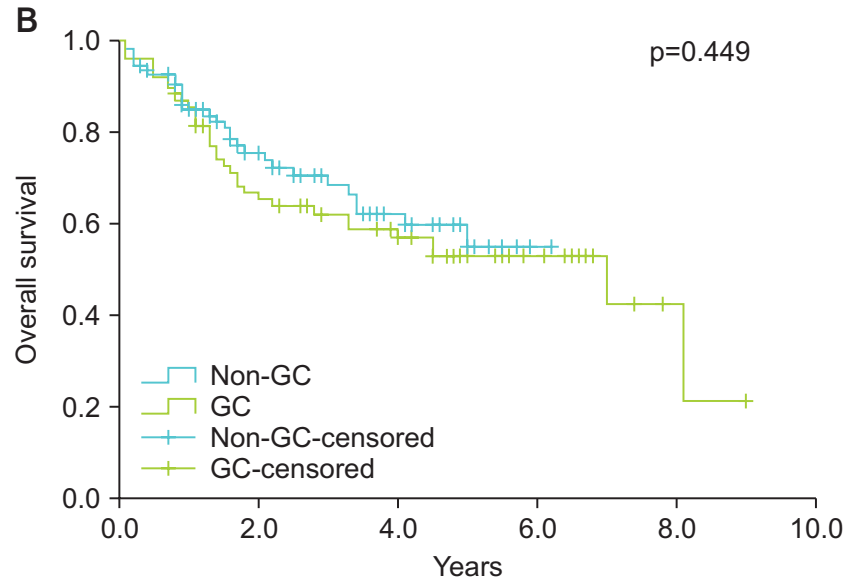

Fig. 2. Kaplan-Meier curves for metastasis-free survival (A) and overall survival (B) of 185 patients with bladder cancer undergoing radical cystectomy, stratified through perioperative administration of glucocorticoid (GC). (C) Kaplan-Meier curves for metastasis-free survival of 30 patients with bladder cancer undergoing radical cystectomy in the nontransfusion subgroup after propensity score matching, stratified through perioperative administration of GC.

Table 2. Univariate Cox regression analyses for distant MFS and OS

\begin{tabular}{|c|c|c|c|c|}
\hline \multirow{2}{*}{ Variable } & \multicolumn{2}{|c|}{ MFS univariate analysis } & \multicolumn{2}{|c|}{ OS univariate analysis } \\
\hline & HR $(95 \% \mathrm{Cl})$ & $p$-value & HR $(95 \% \mathrm{Cl})$ & p-value \\
\hline Age at surgery & $1.034(1.007-1.061)$ & 0.012 & $1.044(1.018-1.071)$ & 0.001 \\
\hline Sex (reference: female) & $1.437(0.618-3.339)$ & 0.400 & $1.175(0.535-2.579)$ & 0.688 \\
\hline \multicolumn{5}{|l|}{ Pathologic T stage } \\
\hline$\geq \mathrm{T} 3$ & $2.599(1.567-4.313)$ & $<0.001$ & $2.214(1.352-3.626)$ & 0.002 \\
\hline$\leq \mathrm{T} 2$ & Reference & & Reference & \\
\hline \multicolumn{5}{|l|}{ Nodal status } \\
\hline Positive & $2.511(1.3526-4.663)$ & 0.004 & $3.233(1.841-5.679)$ & $<0.001$ \\
\hline Negative & Reference & & Reference & \\
\hline Tumor grade (high vs. low) & $0.958(0.376-2.442)$ & 0.928 & $1.508(0.534-4.264)$ & 0.438 \\
\hline Tumor size $(\geq 3 \mathrm{~cm}$ vs. $<3 \mathrm{~cm})$ & $1.422(0.828-2.442)$ & 0.202 & $1.023(0.620-1.687)$ & 0.930 \\
\hline Number of tumors (multiple vs. single) & $0.787(0.5474-1.306)$ & 0.354 & $0.683(0.417-1.117)$ & 0.129 \\
\hline Prior recurrence history & $1.192(0.724-1.962)$ & 0.490 & $0.992(0.608-1.618)$ & 0.975 \\
\hline Concurrent CIS (yes vs. no) & $0.449(0.110-1.840)$ & 0.266 & $0.045(0.001-3.476)$ & 0.162 \\
\hline Adjuvant therapy (yes vs. no) & $1.511(0.831-2.746)$ & 0.176 & $1.546(0.877-2.723)$ & 0.132 \\
\hline \multicolumn{5}{|l|}{ Perioperative blood transfusion } \\
\hline Yes & $1.805(1.075-3.031)$ & 0.025 & $1.640(0.988-2.724)$ & 0.056 \\
\hline No & Reference & & Reference & \\
\hline \multicolumn{5}{|l|}{ Glucocorticoid } \\
\hline Yes & $1.980(1.181-3.321)$ & 0.010 & $1.185(0.721-1.946)$ & 0.503 \\
\hline No & Reference & & Reference & \\
\hline
\end{tabular}

MFS, metastasis-free survival; OS, overall survival; $\mathrm{HR}$, hazard ratio; $\mathrm{Cl}$, confidence interval; $\mathrm{CIS}$, carcinoma in situ . 
Table 3. Multivariate analysis of clinicopathological factors associated with MFS

\begin{tabular}{|c|c|c|c|c|c|c|}
\hline \multirow{2}{*}{ Variable } & \multicolumn{2}{|l|}{ MFS } & \multicolumn{2}{|l|}{ MFS } & \multicolumn{2}{|l|}{ MFS } \\
\hline & $\mathrm{HR}(95 \% \mathrm{CI})$ & $p$-value & $\mathrm{HR}(95 \% \mathrm{Cl})$ & p-value & $\mathrm{HR}(95 \% \mathrm{CI})$ & p-value \\
\hline Age (y) & $1.026(0.998-1.055)$ & 0.066 & $1.028(1.000-1.056)$ & 0.049 & $1.026(0.999-1.055)$ & 0.062 \\
\hline \multicolumn{7}{|l|}{ Tumor stage } \\
\hline$\geq \mathrm{T} 3$ & $2.088(1.180-3.697)$ & 0.011 & $2.055(1.163-3.632)$ & 0.013 & $2.106(1.194-3.714)$ & 0.010 \\
\hline$\leq \mathrm{T} 2$ & Reference & & Reference & & Reference & \\
\hline \multicolumn{7}{|l|}{ Nodal status } \\
\hline Positive & $1.608(0.795-3.251)$ & 0.001 & $1.550(0.769-3.122)$ & 0.220 & $1.709(0.856-3.412)$ & 0.129 \\
\hline Negative & Reference & & Reference & & Reference & \\
\hline \multicolumn{7}{|c|}{ Intraoperative blood transfusion } \\
\hline Yes & $1.389(0.715-2.696)$ & 0.332 & & & $1.751(1.036-2.960)$ & 0.036 \\
\hline No & Reference & & & & Reference & \\
\hline \multicolumn{7}{|l|}{ Glucocorticoid } \\
\hline Yes & $1.462(0.754-2.836)$ & 0.261 & $1.790(1.058-3.028)$ & 0.030 & & \\
\hline No & Reference & & Reference & & & \\
\hline
\end{tabular}

MFS, metastasis-free survival; $\mathrm{HR}$, hazard ratio; $\mathrm{Cl}$, confidence interval.

transfusion subgroup, GCs showed a nonsignificant tendency to increase the risk of metastasis in BC. To the best of our knowledge, the present study is the first to investigate the effect of perioperative GCs on postoperative metastasis and mortality in patients with $\mathrm{BC}$, and we found that perioperative GCs might have an increased risk of distant metastasis; this finding suggests that GC should be used with caution in perioperative morbidity management in patients undergoing $\mathrm{BC}$ surgery. For patients with nausea and vomiting, allergies, fever, and a need for blood transfusion, alternative drugs such as central antiemetics, nonsteroidal antipyretics, and antiallergic $\mathrm{H} 1$ blockers can be used.

Dexamethasone is an effective and widely used GC that is administered perioperatively to improve the quality of recovery after surgery. However, dexamethasone can also induce immunosuppression and may cause tumor cells to escape immune surveillance. From this perspective, it is very important to investigate the effects of perioperative dexamethasone administration on postoperative oncologic outcomes in patients who are already immunocompromised due to the perioperative environment. A retrospective study of 515 patients who underwent colon cancer surgery found that patients with low-dose dexamethasone during the perioperative period had a higher risk of recurrence, and in the nontransfusion subgroup, GC remained as an independent risk factor for recurrence and OS [15]. Moreover, a previous randomized clinical trial found that preoperative single-dose dexamethasone use potentially increased the risk of distant recurrence in patients undergoing colon cancer surgery [16]. On the other hand, given the putative immunosuppressive and antitumor effects, several recent studies have investigated the association be- tween the intraoperative use of dexamethasone and survival after cancer surgery. In patients who underwent non-small cell lung cancer surgery, the intraoperative use of dexamethasone was not significantly associated with recurrence-free survival and OS [17]. In female patients with ovarian cancer, intraoperative dexamethasone administration did not affect postoperative survival; in contrast, perioperative dexamethasone administration was associated with longer survival after pancreatic cancer surgery [18,19]. A recent retrospective study of 2,623 patients who underwent breast cancer surgery showed that a perioperative single dose of dexamethasone did not significantly increase recurrence or mortality of patients with breast cancer. Subgroup analyses, however, revealed that dexamethasone showed a significant tendency to increase postoperative mortality in patients with progesterone receptor-positive breast cancer. Moreover, dexamethasone was significantly associated with the risk of recurrence in TNM stage I of breast cancer [20]. These studies suggest that GCs may play different roles in postoperative tumor survival for different tumor types and specific subpopulations of certain tumors.

In a population-based case-control study that included $786 \mathrm{BC}$ cases and 1,083 controls, the results showed that systemic use of GCs was associated with an increased risk of BC; a further subgroup analysis showed that GC use was significantly associated with more advanced disease stage and grade and higher odds of having TP53 high-intensity stained tumors. It is well known that surgical procedures increase the probability of tumor cells entering the bloodstream [21]. At the same time, the stress response caused by surgery can lead to changes in the body's immunological, 
endocrinological, and hematological systems, which may facilitate the proliferation and customization of tumor cells [22]. GCs are widely used for the treatment of perioperative complications because the GC receptor mediates the effects of stress hormones. GC shows both indirect and direct effects on tumor cells. On the one hand, pharmacological properties of dexamethasone itself, such as immunosuppression and anti-inflammatory properties, may indirectly contribute to the survival of tumor cells [23]. On the other hand, previous studies have shown that GCs can directly inhibit the proliferation, invasion, and migration of tumor cells, such as colon cancer cells and breast cancer cells [24,25]. However, other preclinical research studies have demonstrated that GCs in vitro can promote the proliferation of breast cancer cells [8], oral carcinoma cells [26], and prostate cancer cells [27]. The underlying molecular mechanisms by which GCs affect BC through the GR pathway, however, remain poorly understood. In particular, it is notable that GCs have been shown to strongly inhibit migration and invasion of $\mathrm{BC}$ cells, but promote cell proliferation and reduce apoptosis [28]. In addition, dexamethasone treatment of mouse fibroblasts has been shown to increase GR beta (GRß), which is a hormoneinsensitive dominant-negative inhibitor of GC receptor alpha [29]. Furthermore, McBeth et al. [30] found that elevated GR $\beta$ promotes migration of human $\mathrm{BC}$ cells. Recently, Obradović et al. [7] demonstrated that GCs can promote tumor colonization and metastasis by activating GR signaling pathways in breast cancer. Hence, these results suggest that the association of perioperative GCs with a higher risk of metastasis can possibly be explained by indirect systemic immunosuppression and direct activation of GR signaling pathways in BC.

The present study has several limitations. First, the study is limited by its retrospective nature and selection bias, and the effects of unmeasured confounding variables cannot be excluded. Second, the patients were administered GC during surgery depending on the opinion of the anesthesiologist, and most of them were also given blood transfusion. On the other hand, GC use after surgery might have been influenced by factors such as transfusion and comorbidities that might be considered as sources of bias. Third, this was a single-center study and the follow-up time was not long enough to meet all evaluation endpoints. Therefore, the results must be interpreted with caution.

\section{CONCLUSIONS}

Perioperative GCs were associated with a higher rate of distant metastasis in patients undergoing RC for BC. Given a significant positive correlation between perioperative GC and blood transfusion, this finding warrants further investigation in a subgroup of patients without perioperative blood transfusion.

\section{CONFLICTS OF INTEREST}

The authors have nothing to disclose.

\section{ACKNOWLEDGMENTS}

This study was funded by the Shanghai Science Committee Foundation (grant number 19411967700) and Natural Science Foundation of China (grant number 81472389, 81672549).

\section{AUTHORS' CONTRIBUTIONS}

Research conception and design: Xudong Yao. Data acquisition: Shiyu Mao, Yuan Wu, and Jing Yuan. Statistical analysis: Ruiliang Wang, Yadong Guo, and Wentao Zhang. Data analysis and interpretation: Shiyu Mao, Yuan Wu, and Jing Yuan. Drafting of the manuscript: Shiyu Mao. Critical revision of the manuscript: Junfeng Zhang, Yang Yan, and Xudong Yao. Obtaining funding: Xudong Yao. Administrative, technical, or material support: Xudong Yao. Supervision: Junfeng Zhang, Yang Yan, and Xudong Yao. Approval of the final manuscript: Shiyu Mao, Yuan Wu, Ruiliang Wang, Yadong Guo, Jing Yuan, Wentao Zhang, Junfeng Zhang, Yang Yan, and Xudong Yao.

\section{SUPPLEMENTARY MATERIAL}

Scan this QR code to see the supplementary material, or visit https://www.icurology.org/src/sm/icurology-61-382-s001.pdf.

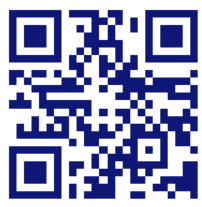

\section{REFERENCES}

1. Richters A, Aben KKH, Kiemeney LALM. The global burden of urinary bladder cancer: an update. World J Urol 2019 Nov 1 [Epub]. https://doi.org/10.1007/s00345-019-02984-4.

2. Blaschke S, Koenig F, Schostak M. Hematogenous tumor cell spread following standard transurethral resection of bladder carcinoma. Eur Urol 2016;70:544-5. 
3. Koch M, Kienle P, Hinz U, Antolovic D, Schmidt J, Herfarth $\mathrm{C}$, et al. Detection of hematogenous tumor cell dissemination predicts tumor relapse in patients undergoing surgical resection of colorectal liver metastases. Ann Surg 2005;241:199-205.

4. Snyder GL, Greenberg S. Effect of anaesthetic technique and other perioperative factors on cancer recurrence. Br J Anaesth 2010;105:106-15.

5. Gündisch S, Boeckeler E, Behrends U, Amtmann E, Ehrhardt $\mathrm{H}$, Jeremias I. Glucocorticoids augment survival and proliferation of tumor cells. Anticancer Res 2012;32:4251-61.

6. Zhang C, Kolb A, Mattern J, Gassler N, Wenger T, Herzer K, et al. Dexamethasone desensitizes hepatocellular and colorectal tumours toward cytotoxic therapy. Cancer Lett 2006;242:104-11.

7. Obradović MMS, Hamelin B, Manevski N, Couto JP, Sethi A, Coissieux MM, et al. Glucocorticoids promote breast cancer metastasis. Nature 2019;567:540-4.

8. Sorrentino G, Ruggeri N, Zannini A, Ingallina E, Bertolio R, Marotta C, et al. Glucocorticoid receptor signalling activates YAP in breast cancer. Nat Commun 2017;8:14073.

9. Arora VK, Schenkein E, Murali R, Subudhi SK, Wongvipat J, Balbas MD, et al. Glucocorticoid receptor confers resistance to antiandrogens by bypassing androgen receptor blockade. Cell 2013;155:1309-22.

10. Boström PJ, Kössi J, Laato M, Nurmi M. Risk factors for mortality and morbidity related to radical cystectomy. BJU Int 2009;103:191-6.

11. Rosenberg JE, Carroll PR, Small EJ. Update on chemotherapy for advanced bladder cancer. J Urol 2005;174:14-20.

12. Bajorin DF, Dodd PM, Mazumdar M, Fazzari M, McCaffrey JA, Scher HI, et al. Long-term survival in metastatic transitional-cell carcinoma and prognostic factors predicting outcome of therapy. J Clin Oncol 1999;17:3173-81.

13. Abel EJ, Linder BJ, Bauman TM, Bauer RM, Thompson RH, Thapa $\mathrm{P}$, et al. Perioperative blood transfusion and radical cystectomy: does timing of transfusion affect bladder cancer mortality? Eur Urol 2014;66:1139-47.

14. Linder BJ, Frank I, Cheville JC, Tollefson MK, Thompson RH, Tarrell RF, et al. The impact of perioperative blood transfusion on cancer recurrence and survival following radical cystectomy. Eur Urol 2013;63:839-45.

15. Yu HC, Luo YX, Peng H, Kang L, Huang MJ, Wang JP. Avoiding perioperative dexamethasone may improve the outcome of patients with rectal cancer. Eur J Surg Oncol 2015;41:667-73.

16. Singh PP, Lemanu DP, Taylor MH, Hill AG. Association between preoperative glucocorticoids and long-term survival and cancer recurrence after colectomy: follow-up analysis of a previous randomized controlled trial. Br J Anaesth 2014;113 Suppl 1:i68-73.

17. Cata JP, Jones J, Sepesi B, Mehran RJ, Rodriguez-Restrepo A,
Lasala J, et al. Lack of association between dexamethasone and long-term survival after non-small cell lung cancer surgery. J Cardiothorac Vasc Anesth 2016;30:930-5.

18. De Oliveira GS Jr, McCarthy R, Turan A, Schink JC, Fitzgerald $\mathrm{PC}$, Sessler DI. Is dexamethasone associated with recurrence of ovarian cancer? Anesth Analg 2014;118:1213-8.

19. Call TR, Pace NL, Thorup DB, Maxfield D, Chortkoff B, Christensen J, et al. Factors associated with improved survival after resection of pancreatic adenocarcinoma: a multivariable model. Anesthesiology 2015;122:317-24.

20. Kim MH, Kim DW, Park S, Kim JH, Lee KY, Hwang J, et al. Single dose of dexamethasone is not associated with postoperative recurrence and mortality in breast cancer patients: a propensity-matched cohort study. BMC Cancer 2019;19:251.

21. Dietrich K, Schned A, Fortuny J, Heaney J, Marsit C, Kelsey $\mathrm{KT}$, et al. Glucocorticoid therapy and risk of bladder cancer. Br J Cancer 2009;101:1316-20.

22. Desborough JP. The stress response to trauma and surgery. Br J Anaesth 2000;85:109-17.

23. Shakhar G, Blumenfeld B. Glucocorticoid involvement in suppression of NK activity following surgery in rats. J Neuroimmunol 2003;138:83-91.

24. Kim JH, Hwang YJ, Han SH, Lee YE, Kim S, Kim YJ, et al. Dexamethasone inhibits hypoxia-induced epithelial-mesenchymal transition in colon cancer. World J Gastroenterol 2015;21:9887-99.

25. Buxant F, Kindt N, Laurent G, Noël JC, Saussez S. Antiproliferative effect of dexamethasone in the MCF-7 breast cancer cell line. Mol Med Rep 2015;12:4051-4.

26. Xie H, Li C, He Y, Griffin R, Ye Q, Li L. Chronic stress promotes oral cancer growth and angiogenesis with increased circulating catecholamine and glucocorticoid levels in a mouse model. Oral Oncol 2015;51:991-7.

27. Puhr M, Hoefer J, Eigentler A, Ploner C, Handle F, Schaefer G, et al. The glucocorticoid receptor is a key player for prostate cancer cell survival and a target for improved antiandrogen therapy. Clin Cancer Res 2018;24:927-38.

28. Zheng Y, Izumi K, Li Y, Ishiguro H, Miyamoto H. Contrary regulation of bladder cancer cell proliferation and invasion by dexamethasone-mediated glucocorticoid receptor signals. Mol Cancer Ther 2012;11:2621-32.

29. Hinds TD Jr, Ramakrishnan S, Cash HA, Stechschulte LA, Heinrich G, Najjar SM, et al. Discovery of glucocorticoid receptor-beta in mice with a role in metabolism. Mol Endocrinol 2010;24:1715-27.

30. McBeth L, Nwaneri AC, Grabnar M, Demeter J, NestorKalinoski A, Hinds TD Jr. Glucocorticoid receptor beta increases migration of human bladder cancer cells. Oncotarget 2016;7:27313-24. 